\title{
ON THE $w^{*}$-SEQUENTIAL CLOSURE OF A CONE
}

\author{
R. D. McWILliamS
}

1. If $X$ is a real Banach space and $X^{* *}$ the second conjugate space of $X$, then for each subset $A$ of $X^{* *}$ let $K_{X}(A)$ be the $w^{*}$-sequential closure of $A$ in $X^{* *}$; thus $F \in K_{X}(A)$ if and only if there is a sequence $\left\{F_{n}\right\}$ in $A$ such that $F(f)=\lim _{n} F_{n}(f)$ for all $f \in X^{*}$. If $J_{X}$ is the canonical mapping from $X$ into $X^{* *}$, then $K_{X}\left(J_{X} X\right)$ is closed in the norm topology of $X^{* *}[1]$. In the present paper it will be shown that if $P$ is a norm-closed cone in $X$, then $K_{X}\left(J_{X} P\right)$ is norm-closed in $X^{* *}$, but $K_{X}\left(K_{X}\left(J_{X} P\right)\right)$ need not be norm-closed in $X^{* *}$.

2. If $s$ is a bounded real function on $[0,1]$, let $\|s\|=\sup _{0 \leq t \leq 1}|s(t)|$. If $S=\left\{s_{k}\right\}$ is a bounded sequence of functions, i.e., if each $s_{k}$ is bounded and $\sup _{k \in \omega}\left\|s_{k}\right\|$ is finite, let $\|S\|=\sup _{k \in \omega}\left\|s_{k}\right\|$. If $Q$ is a set of functions, let $L(Q)$ be the set of all functions $x$ such that $x$ is the pointwise limit of a bounded sequence in $Q$. Let $a$ be the collection of all double sequences $A=\left\{a_{k i}\right\}$ of non-negative numbers such that for each $k$ the following conditions are satisfied: (1) $a_{k i}=0$ for all $i<k$ and for all but a finite number of $i \geqq k$; and (2) $\sum_{i} a_{k i}=1$. If $S=\left\{s_{k}\right\}$ is a sequence of functions and $A \in Q$, let $T_{\Delta} S$ be the sequence $\left\{s_{k}^{\prime}\right\}$ such that $s_{k}^{\prime}=\sum_{i} a_{k i} s_{i}$ for each $k$. It is clear that (1) $T_{A}$ is a linear operator; (2) if $S$ is bounded, then so is $T_{A} S$ and $\left\|T_{A} S\right\| \leqq\|S\| ;$ (3) if $S$ is pointwise convergent, then $T_{A} S$ is pointwise convergent to the same limit.

LEMMA 1. If $R$ and $S$ are bounded sequences in the space $\mathfrak{e}[0,1]$ of continuous real functions on the interval $[0,1]$ and $R$ and $S$ converge pointwise to functions $r$ and $s$ respectively, then for each $\epsilon>0$ there exists $A \in$ a such that $\left\|T_{A} R-T_{A} S\right\|<\|r-s\|+\epsilon$.

Proof. The sequence $R-S$ is bounded and converges pointwise to $r-s$. Hence by $[1$; proof of Lemma 1] there exists for each $\epsilon>0$ an $A$ having the required property.

Lemma 2. Let $P$ be a cone in $\mathcal{C}[0,1]$, let $\left\{z_{n}\right\}$ be a sequence in $L(P)$, and let $z$ be a bounded function such that $\lim _{n \rightarrow \infty}\left\|z_{n}-z\right\|=0$. Then $z \in L(P)$.

Proof. It may be assumed that $\left\|z_{n}-z\right\|<2^{-n}$ for each $n$. For each

Presented to the Society, 'February 22, 1962 under the title $w^{*}$-sequential closure of a cone in a Banach space; received by the editors December 4, 1961. 
$n$ there is a bounded sequence $S_{n}=\left\{s_{n k}\right\}$ in $P$ which converges pointwise to $z_{n}$; by Lemma 1 it may be assumed that $\left\|S_{n}\right\|<\|z\|+2^{-n}$.

By induction on $n$ it can now be shown that for each $n$ and for each $i \leqq n$ there exists a bounded sequence $S_{i n}=\left\{s_{i n k}\right\}$ in $P$ such that

and

$$
\begin{array}{ll}
\lim _{k \rightarrow \infty} s_{i n k}(t)=z_{i}(t) & \text { for each } t \in[0,1], \\
\lim _{k \rightarrow \infty} s_{i k k}(t)=z_{i}(t) & \text { for each } t \in[0,1],
\end{array}
$$

$$
\left\|S_{i n}-S_{j n}\right\|<2^{1-i} \quad \text { if } i<j \leqq n .
$$

For $n=1$, the sequence $S_{11}$ can be taken to be $S_{1}$. For $n>1$, if $S_{i, n-1}$ has been obtained for each $i \leqq n-1$, Lemma 1 may be applied $(n-1)$ times in succession to obtain $A_{1}, \cdots, A_{n-1} \in Q$ such that

$$
\left\|T_{A_{i}} T_{A_{i-1}} \cdots T_{A_{1}}\left(S_{i, n-1}-S_{n}\right)\right\|<2^{1-i}
$$

for each $i<n$. The induction step is completed by letting $S_{\text {in }}$ $=T_{A_{n-1}} \cdots T_{A_{1}} S_{i, n-1}$ for each $i<n$ and $S_{n n}=T_{A_{n-1}} \cdots T_{A_{1}} S_{n}$.

Now let $S=\left\{s_{k}\right\}$ be the bounded sequence in $P$ such that $s_{k}=s_{k k k}$ for each $k$. Let $t \in[0,1]$ and $i \in \omega$. Then for every $k \geqq i$,

$$
\begin{aligned}
& \left|s_{k}(t)-z(t)\right| \\
& \quad \leqq\left|s_{k k k}(t)-s_{i k k}(t)\right|+\left|s_{i k k}(t)-z_{i}(t)\right|+\left|z_{i}(t)-z(t)\right| \\
& \quad<3 \cdot 2^{-i}+\left|s_{i k k}(t)-z_{i}(t)\right| .
\end{aligned}
$$

Since by construction $\lim _{k \rightarrow \infty} s_{i k k}(t)=z_{i}(t)$, it follows that $\lim _{k \rightarrow \infty} s_{k}(t)$ $=z(t)$ for every $t$, so that $z \in L(P)$.

TheOREM 1. If $P$ is a norm-closed cone in a real Banach space $X$, then $K_{X}\left(J_{X} P\right)$ is norm-closed in $X^{* *}$.

Proof. Let $F \in X^{* *}$ be the limit in norm of a sequence $\left\{F_{n}\right\}$ $\subset K_{X}\left(J_{X} P\right)$. Thus each $F_{n}$ is the $w^{*}$-limit of a bounded [3, p. 209] sequence $J_{X} S_{n}$, where $S_{n}=\left\{s_{n k}\right\} \subset P$.

CASE 1. First suppose that $X$ is a closed subspace of $\mathfrak{e}[0,1]$. For each $t \in[0,1]$ let $f_{t} \in X^{*}$ be defined by $f_{t}(s)=s(t)$ for all $s \in X$. Then for each $n$ it follows that $F_{n}\left(f_{t}\right)=\lim _{k \rightarrow \infty} s_{n k}(t)$. If $z_{n}$ is defined on $[0,1]$ by $z_{n}(t)=F_{n}\left(f_{t}\right)$, it follows that $z_{n} \in L(P)$ and moreover, since $\left\|f_{t}\right\| \leqq 1$ for each $t$, that $\left\|z_{n}-z_{m}\right\| \leqq\left\|F_{n}-F_{m}\right\|$ for all $n$ and $m$, so that the function $z(t)=\lim _{n \rightarrow \infty} z_{n}(t)$ exists and belongs to $L(P)$ by Lemma 2. Thus there is a bounded sequence $\left\{s_{k}\right\} \subset P$ which converges pointwise to $z$. Since $X$ is a subspace of $\mathfrak{e}[0,1]$, for each $f \in X^{*}$ there is a finite regular signed Borel measure $\mu_{f}$ on $[0,1]$ such that $f(x)$ 
$=\int_{0}^{1} x d \mu_{f}$ for every $x \in X[3$, p. 397]. Hence

$$
F_{n}(f)=\lim _{k \rightarrow \infty} f\left(s_{n k}\right)=\int_{0}^{1} z_{n} d \mu_{f}
$$

for every $n$, and therefore

$$
F(f)=\lim _{n} \int_{0}^{1} z_{n} d \mu_{f}=\int_{0}^{1} z d \mu_{f}=\lim _{k \rightarrow \infty} \int_{0}^{1} s_{k} d \mu_{f}=\lim _{k \rightarrow \infty} f\left(s_{k}\right),
$$

so that $F$ is the $w^{*}$-limit of $\left\{J_{X} s_{k}\right\}$.

CASE 2. If $X$ is an arbitrary real Banach space, let $Y$ be the normclosed subspace and $Q$ the norm-closed cone in $X$ generated by $\left\{s_{n k}: n, k \in \omega\right\}$. Since $Y$ is separable, there is an equivalence mapping $E$ from $Y$ onto a closed subspace $Z$ of $\mathfrak{e}[0,1]$. Since each $F_{n} \in K_{X}\left(J_{X} P\right)$, for each $n$ an element $G_{n} \in Y^{* *}$ is unambiguously defined by

$$
G_{n}(f \mid Y)=F_{n}(f) \quad \text { for all } f \in X^{*} .
$$

The sequence $\left\{G_{n}\right\}$ is clearly Cauchy since each $g \in Y^{*}$ has an extension $f \in X^{*}$ such that $\|f\|=\|g\|$. Since $\left\{G_{n}\right\} \subset K_{Y}(Q)$, it follows that the sequence $\left\{E^{* *} G_{n}\right\}$ is a Cauchy sequence in $K_{Z}\left(J_{Z} E Q\right)$ converging in norm to $E^{* *} G$. By Case 1 there is a bounded sequence $\left\{z_{k}\right\}$ $C E Q$ such that $\left\{J_{Z z_{k}}\right\}$ is $w^{*}$-convergent to $E^{* *} G$ in $Z^{* *}$. Finally, it is straightforward to verify that the sequence $\left\{J_{X} E^{-1} z_{k}\right\} \subset J_{X} P$ is $w^{*}$-convergent to $F$ in $X^{* *}$.

3. For each $t_{o} \in[0,2]$ and $q \in \omega$ let $f_{t_{0} q} \in \mathcal{C}[0,2]$ be defined by

$$
f_{t_{0} q}(t)=\max \left[0,1-2^{q}\left|t-t_{0}\right|\right] \quad \text { for } t \in[0,2] .
$$

If $p, i$, and $j$ are positive integers, let $s_{p i}=2^{-p} i$ and $t_{j}=2-2^{-i}$. Now if $a>1$ let $X^{a}$ be the norm-closed subspace of $\mathfrak{e}[0,2]$ generated by the set $G^{a}=\left\{x_{p q}^{a}: p, q \in \omega\right\}$, where

$$
x_{p q}^{a}(t)=\max \left[\max _{1 \leqq i<2^{p}} f_{s_{p i}, p+q}(t), \max _{p \leqq j<p+q} a f_{t, j+q}(t)\right] .
$$

Lemma 3. If $P^{a}$ is the norm-closed cone in $X^{a}$ generated by $\left\{x_{p q}^{a}: p, q \in \omega\right\}$, then the function $x_{0}$ defined by

$$
x_{0}(t)=\lim _{D \in \omega}\left[\lim _{a \in \omega} x_{p q}^{a}(t)\right]
$$

is an element of $L\left(L\left(P^{a}\right)\right)$ with $\left\|x_{0}\right\|=1$, but if $\left\{y^{h}\right\}$ is a bounded sequence in $L\left(P^{a}\right)$ converging pointwise to $x_{o}$, then $\lim \sup _{h \in \omega}\left\|y^{h}\right\| \geqq a$.

Proof. It is trivial that $x_{0} \in L(L(P))$ and $\left\|x_{0}\right\|=1$. Indeed, $x_{0}$ is 
the characteristic function of the set $D=\left\{s_{p i}: p \in \omega, i<2^{p}\right\}$. If $\left\{y^{h}\right\}$ is an arbitrary bounded sequence in $L\left(P^{a}\right)$ converging pointwise to $x_{o}$, then since the set of all finite linear combinations of the $x_{p e}^{a}$ with non-negative coefficients is dense in $P^{a}$, each $y^{h}$ is the pointwise limit of a bounded sequence $\left\{y^{h k}\right\}_{k=1}^{\infty}$, where each $y^{h k}$ has the form

$$
y^{h k}(t)=\sum_{p \geq 1 ; q \geq 1} a_{p q}^{h k} x_{p q}^{a}(t),
$$

where each $a_{p q}^{h \mathbf{k}}$ is non-negative and for each pair $(h, k)$ only a finite number of the $a_{p q}^{n k}$ are positive. Without changing the value of $\lim \sup _{h \in \omega}\left\|y^{h}\right\|$, it may be assumed that $y^{h k}\left(\frac{1}{2}\right)=1$ for all $(h, k)$.

Let $\epsilon>0$ be given. For each $H \in \omega$ let

$$
S_{H}=\left\{t \in[0,1]: t \notin D, y^{h}(t)<\epsilon \text { for all } h \geqq H\right\} .
$$

Since $U_{H \in \omega} S_{H}$ is of the second category in $[0,1]$, there exists an $H$ such that $S_{H}$ is dense in a closed interval $I$. Choose an arbitrary $s_{p_{0} i_{0}} \in D \cap($ int $I)$ with $i_{o}$ odd; then there exists $H_{0} \geqq H$ such that $y^{h}\left(s_{p_{o} i_{o}}\right)>1-\epsilon$ for all $h \geqq H_{o}$. Since there exist points of $S_{H}$ between $s_{p_{0} i_{o}}$ and every $s_{p i}$ such that $p<p_{o}$, it is clear that for every $h \geqq H_{\text {。 }}$ there exists $K_{h}$ such that for every $k \geqq K_{h}$,

$$
\sum_{p \geq p_{0 ;} ; \geq 1} a_{p q}^{h k}=y^{h k}\left(s_{p_{0} i_{o}}\right)-\sum_{p<p_{o} ; q \geq 1} a_{p q}^{h k} x_{p q}^{a}\left(s_{p_{o} i_{o}}\right)>(1-\epsilon)-2 \epsilon .
$$

Now fix $h \geqq H_{o}$. Since $y^{h}$ is a Baire function of the first class, there exists a point $\sigma_{h} \in I$ such that $y^{h} \mid I$ is continuous at $\sigma_{h}[2$, p. 143]; hence there is a closed interval $J \subset I$ such that $y^{h}(t)<2 \epsilon$ for all $t \in J$. Choose $s_{p_{1} i_{1}} \in D \cap\left(\right.$ int $J$ ) with $p_{1}>p_{o}$; then there exists $K_{h}^{\prime} \geqq K_{h}$ such that for every $k \geqq K_{h}^{\prime}$,

$$
\sum_{p \geq p_{1 ;} ; q \geq 1} a_{p q}^{h k} \leqq y^{h k}\left(s_{p_{1} i_{1}}\right)<2 \epsilon .
$$

Next, by (3.2), for every $t \in[0,1]$ and every $k$,

$$
\begin{aligned}
y^{h k}(t) & \geqq \sum_{p_{0} \leq p<p_{1} ; q<p_{1}} a_{p q}^{h k}\left(1-2^{p+q}\left|t-s_{p_{o} i_{o}}\right|\right) \\
& \geqq \sum_{p_{o} \leq p<p_{1} ; q<p_{1}} a_{p q}^{h k}\left(1-2^{2 p_{1}}\left|t-s_{p_{o_{i}}}\right|\right) .
\end{aligned}
$$

There exists $t_{o} \in S_{H}$ such that $\left|t_{o}-s_{p_{o} i_{o}}\right|<2^{-\left(2 p_{1}+1\right)}$, and there exists $K_{h}^{\prime \prime} \geqq K_{h}^{\prime}$ such that $y^{h k}\left(t_{0}\right)<\epsilon$ whenever $k \geqq K_{h}^{\prime \prime}$. Hence, by (3.8) with $t=t_{0}$ 


$$
\sum_{p_{0} \leq p<p_{1} ; q<p_{1}} a_{p q}^{h k}<\frac{\epsilon}{1-2^{2 p_{1}}\left|t_{0}-s_{p_{o} i_{o}}\right|}<2 \epsilon
$$

for all $k \geqq K_{h}^{\prime \prime}$. Therefore, by (3.6), (3.7), and (3.9),

$$
\sum_{p_{0} \leq p<p_{1} ; q \geq p_{1}} a_{p q}^{h k}>1-7 \epsilon
$$

for all $k \geqq K_{h}^{\prime \prime}$, so that $\left\|y^{h}\right\| \geqq \lim _{k \rightarrow \infty} y^{h k}\left(t_{p_{1}}\right) \geqq a(1-7 \epsilon)$. Since $\epsilon$ may be an arbitrarily small positive number, it follows that lim $\sup _{h \in \omega}$ $\left\|y^{h}\right\| \geqq a$.

THEOREM 2. There exist a norm-closed subspace $X$ of $\mathfrak{C}[0,1]$ and $a$ norm-closed cone $P \subset X$ such that $K_{X}\left(K_{X}\left(J_{X} P\right)\right)$ is not norm-closed in $X^{* *}$.

Proof. For each $r \in \omega$ and each real function $x$ defined on $[0,2]$ let $E_{r} x$ be the function defined on $[0,1]$ by

$$
\left(E_{r} x\right)(t)=\left\{\begin{array}{l}
x\left(2^{r+1}\left[t-2^{-r}\right]\right) \quad \text { if } 2^{-r} \leqq t \leqq 2^{1-r}, \\
0 \text { for all other } t
\end{array}\right.
$$

Recalling the notation of Lemma 3 with $a=2^{2 r}$, observe that $E_{r} \mid X^{2^{2 r}}$ is an equivalence mapping onto a subspace of $\mathfrak{C}[0,1]$, since $x(0)$ $=x(2)=0$ for all $x \in X^{2^{2 r}}$. Let $X$ be the closed subspace and $P$ the closed cone in $\mathcal{C}[0,1]$ generated by $U_{r \in \omega}\left\{E_{r} P^{2^{2 r}}\right\}$. For each $r$ it is clear that $E_{r} x_{0} \in L(L(P))$ and hence that an $F_{r} \in X^{* *}$ can be unambiguously defined by

$$
F_{r}(f)=\int_{0}^{1}\left(E_{r} x_{0}\right) d \mu_{f} \quad \text { for all } f \in X^{*}
$$

then $F_{r} \in K_{X}\left(K_{X}\left(J_{X} P\right)\right)$ and $\left\|F_{r}\right\|=1$. Now let $F=\sum_{r=1}^{\infty} 2^{-r} F_{r}$; thus $F$ belongs to the closure of $K_{X}\left(K_{X}\left(J_{X} P\right)\right)$ in the norm topology.

Suppose $F \in K_{X}\left(K_{X}\left(J_{X} P\right)\right)$. Then there is a bounded sequence $\left\{G_{h}\right\} \subset K_{X}\left(J_{X} P\right)$ whose $w^{*}$-limit is $F$. For each pair $(r, h)$ let $z_{r h}$ be the function on $[0,2]$ defined by

$$
z_{r h}(t)=G_{h}\left(f_{2^{-r}+2^{-1-r} t}\right) \quad \text { for } t \in[0,2],
$$

where $f_{u} \in X^{*}$ is defined by $f_{u}(x)=x(u)$ for $x \in X$ and real $u$. Then $\left\{z_{r h}\right\}_{h=1}^{\infty}$ is a bounded sequence in $L\left(P^{2^{2 r}}\right)$ which converges pointwise on $[0,2]$ to $2^{-r} x_{0}$. By Lemma 3 , lim $\sup _{h \in \omega}\left\|z_{r h}\right\| \geqq 2^{r}$, and hence $\lim \sup _{h \in \omega}\left\|G_{h}\right\| \geqq 2^{r}$. Since $r$ is an arbitrary positive integer, the sequence $\left\{G_{h}\right\}$ is unbounded, which gives a contradiction. Thus $F \notin K_{X}\left(K_{X}\left(J_{X} P\right)\right)$ and the theorem is proved. 
REMARK. The author has not been able to determine whether $K_{\mathbf{X}}\left(K_{\mathbf{X}}\left(J_{\mathbf{X}} X\right)\right)$ can fail to be norm-closed in $X^{* *}$.

\section{REFERENCES}

1. R. D. McWilliams, A note on weak sequential convergence, Pacific J. Math. 12 (1962), 333-335.

2. I. P. Natanson, Theory of functions of a real variable, Vol. II (translated by L. F. Boron), Ungar, New York, 1960.

3. A. E. Taylor, Functional analysis, Wiley, New York, 1958.

Florida State UniverestTy 